\title{
Unusual Tuberculosis of the Frontal Bone: A Case Report
}

\author{
Xiaoyu Wang1,2, Qian Zhang², Wei Wu ${ }^{2 *}$ \\ ${ }^{1}$ School of Clinical Medicine, Xi'an Medical University, Xi'an, China \\ ${ }^{2}$ Department of Respiratory and Critical Care Medicine, The Second Affiliated Hospital of Xi'an Medical University, Xi'an, China \\ Email: *wwatp@163.com
}

How to cite this paper: Wang, X.Y., Zhang, Q. and $\mathrm{Wu}, \mathrm{W}$. (2020) Unusual Tuberculosis of the Frontal Bone: A Case Repor. Open Journal of Internal Medicine, 10, 321-325.

https://doi.org/10.4236/ojim.2020.104033

Received: September 11, 2020

Accepted: October 27, 2020

Published: October 30, 2020

Copyright (๑) 2020 by author(s) and Scientific Research Publishing Inc. This work is licensed under the Creative Commons Attribution International License (CC BY 4.0).

http://creativecommons.org/licenses/by/4.0/

(c) (i) Open Access

\begin{abstract}
Tuberculosis is a chronic infectious disease caused by Mycobacterium tuberculosis bacteria that can affect almost any part of the body, especially in lung. Tuberculosis of the bones is not an unusual condition. However, the occurrence of tuberculosis of the skull is much less compared with the other bones. We report a 19-year-old man exhibiting swelling of the scalp and a bony defect of the skull caused by irregular anti-tuberculosis treatment. He was diagnosed with tuberculosis of right upper lung and underwent the anti-tuberculosis treatment for about 20 days in the local contagious hospital. However, he did not adhere to anti-TB treatment due to feeling better after anti-tuberculosis treatment. He had to receive anti-tuberculous chemotherapy again and underwent surgery after the occurrence of the tuberculosis of frontal bone. Fortunately, the patient completed 9 months of anti-tuberculous therapy and fully recovered. To our knowledge, this is the first report of the tuberculosis of frontal bone caused by irregular anti-tuberculosis treatment.
\end{abstract}

\section{Keywords}

Tuberculosis, Skull, Treatment

\section{Introduction}

Tuberculosis is a chronic infectious disease caused by mycobacterium tuberculosis bacteria that can affect almost any part of the body, especially in lung. Tuberculosis of the bones is not an unusual condition, but the occurrence of tuberculosis of the skull is much less compared with the other bones. The prevalence of calvarial tuberculosis is very rare. Most cases of calvarial tuberculosis occur in young patients. Calvarial tuberculosis is very rare accounting for $0.2 \%$ $1.3 \%$ of skeletal tuberculosis [1]. Herein we report the case of a 19-year-old man 
with tuberculosis which developed into tuberculosis of the frontal owing to irregular anti-tuberculosis treatment.

\section{Case Presentation}

A 19-year-old man was admitted to our hospital because of progressive left frontal swelling, epilepsia and chronic cough for about 1 month. Six months prior to this, he was diagnosed with tuberculosis of right upper lung and underwent the anti-tuberculosis treatment for about 20 days in the local contagious hospital. However, he did not adhere to anti-TB treatment due to his feeling better after anti-tuberculosis treatment. The entire duration of treatment was about 40 days. After about 1 month, he began to cough with night sweat without sputum. Due to little effect on his life, he neglected it and refused therapy. $\mathrm{He}$ did not have a history of diabetes or alcohol consumption. There was no history of TB in either his past or his family.

Concerning his physical examination, his axillary temperature was $37.7^{\circ} \mathrm{C}$, respiration rate 21 breaths/min, and pulse rate 82 beats/min, blood pressure $106 / 63 \mathrm{mmHg}$. On the left frontal region, there was a soft, painless swelling of 4 $\times 6 \mathrm{~cm}$ in diameter. No sinus or erythema was noticed on the lesion (Figure 1). Other systems were normal and no abnormal findings were found on neurological assessment. Laboratory work-up showed that white blood cell count $5280 / \mathrm{mm}^{3}$, platelet count $266,000 / \mathrm{mm}^{3}$, haematocrit level $40 \%$, erythrocyte sedimentation rate $24 \mathrm{~mm} / \mathrm{h}$ and the c-reaction protein level $48.8 \mathrm{mg} / \mathrm{L}$. The patient's urinalysis was normal. Hepatic and nephric function were all normal. The Mantoux test was positive with induration of $17 \mathrm{~mm} \times 19 \mathrm{~mm}$. Polymerase chain reaction (PCR) was positive for tuberculosis, and direct smear was positive for acid fast bacillus (AFB). Head computerized tomography (CT) showed localized swelling of the scalp and a lytic lesion of the skull (Figure 2). CT scan of chest showed miliary nodules diffusely distributed in double lung and a thicken-wall cavity in right lung (Figure 3).

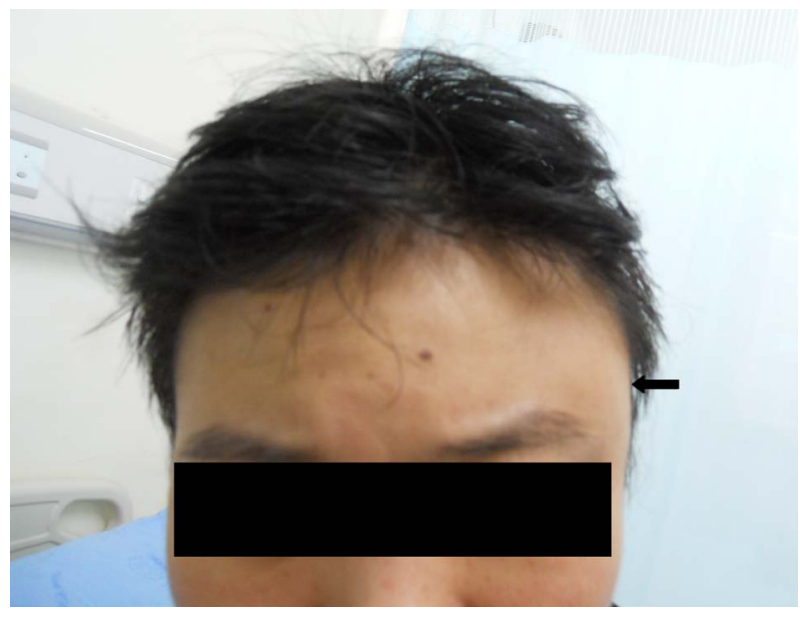

Figure 1. An irregular mass on the left forehead (black arrow). The size is about $4 \times 6 \mathrm{~cm}$. 


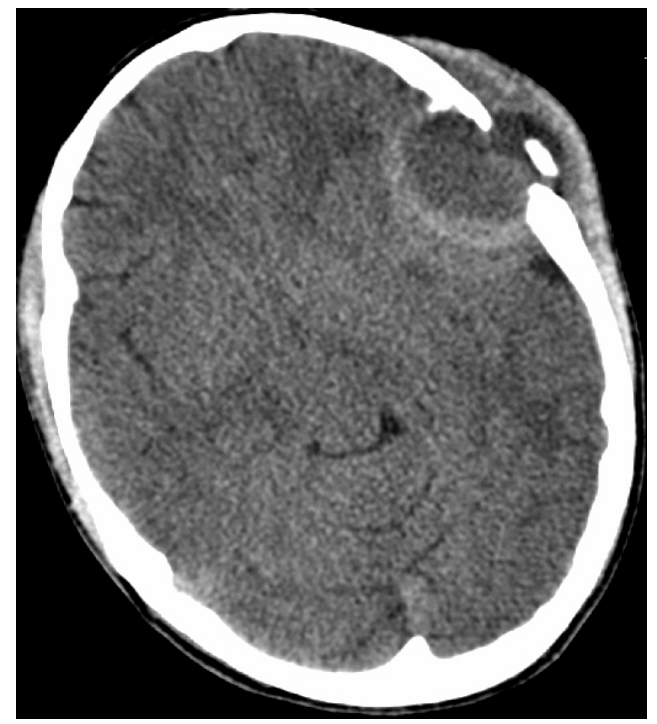

Figure 2. CT scan of the cranium showing localized swelling of the scalp and destruction of the skull.

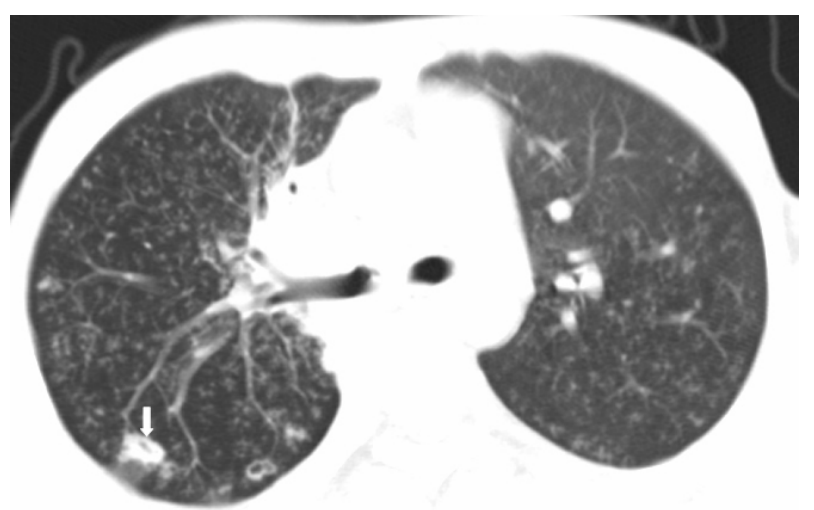

Figure 3. CT scan of chest showing diffuse distribution in double lungs and a thicken-wall cavity in right lung.

In light of the aforementioned findings, a diagnosis of pulmonary TB and secondary skull tuberculosis were made. Antitubercular treatment consisting of isoniazid, rifampin, pyrazinamide, and ethambutol was initiated and the skull abscesses were aspirated.

After nine months of antitubercular treatment, the fever resolved, the abscesses healed, and the patient gained $8 \mathrm{~kg}$ of weight. Follow-up imaging showed resolution of all of lesions previously mentioned.

\section{Discussion}

Up to now, tuberculosis has still been one of health-threatening diseases in developing countries and has produced an enormous social and economic impact, such as in India, China and so on. Tuberculosis of the bones is a common condition, but tuberculosis of the flat bones, particularly those of the frontal, is, though an uncommon condition, by no means a rare one, which usually 
presents as a painless scalp swelling [2]. In 1885, W. Edmunds reported the first tuberculosis of the skull [3]. In addition, the parietal bones are most commonly involved. About seventy-five percent of the cases have occurred in patients under twenty years of age, and the incidence decreases as age increases [4]. However, skull tuberculosis of the elderly also was reported [5].

Due to trauma, which is believed to be an etiological factor, probably, males are more commonly affected. The lungs, the lymphatics, and bones other than the flat bones are the common foci. The posterior cervical lymph glands are most frequently the primary focus, the tonsils being the portal of entry. Infection is usually blood borne from distant foci, and the initial localization is in the cancellous bone of the diploe. The frontal and parietal bones are most commonly affected. Since the flat bones of the vault of the skull contain only a small amount of cancellous bone, it is meaningful to explore the rarity of such lesions [6]. In the majority of cases the process extends first to the inner table of the skull, then to the outer table, and finally perforation occurs, with or without sequestration, depending upon the rapidity of bone destruction. A cold abscess forms at the site of the perforation, and the soft parts, which become elevated by pressure and may rupture spontaneously. Subsequently, fistula formation occurs. These lesions may be single or multiple, but they are always a part of the whole disease process, and single perforations are more commonly found [7].

Extrapulmonary TB is defined as the occurrence of TB at sites other than the lungs, such as the lymph nodes (19\%), pleura (7\%), gastrointestinal tract (4\%), bone and joints (6\%) [8]. Disseminated TB is defined as a TB infection involving the bloodstream, bone marrow, or two or more noncontiguous sites or systems and includes miliary TB involving the lungs. Disseminated TB has been observed for centuries, but its exact incidence is not known [9]. The irregular treatment of tuberculosis caused Mycobacterium tuberculosis to enter the bloodstream from the lungs to the skull, leading to the occurrence of skull tuberculosis abscess. The initial symptoms of skull tuberculosis are usually vague. Generally, localized tenderness and headache are present, followed by swelling of the scalp. For this reason, diagnosis is not made until perforation of the scalp swelling has occurred. The findings are characteristic. We found that the diaphyses of the long bones are not usually infected with tuberculosis. Based on this we attribute the initial focus to the cancellous and flat bone, which usually showed tuberculous osteomyelitis [10].

\section{Conclusion}

Tuberculosis is still a great health-threatening disease and caused millions of deaths each year in the world, especially in developing countries. For various reasons, patients failing to abide by the protocol of anti-TB treatment are not uncommon. Importantly, we should instruct patients to improve their treatment compliance and better their adherence to therapeutic regimen, thus to effectively reduce complications and save their life. 


\section{Conflicts of Interest}

The authors declare no conflicts of interest regarding the publication of this paper.

\section{References}

[1] Bhoi, S.K., Naik, S., Jha, M. and Pradhan, N. (2020) Skull Base and Calvarial Tuberculosis Presenting as Chronic Meningitis. Indian Journal of Tuberculosis, 67, 411-413. https://doi.org/10.1016/j.ijtb.2020.01.011

[2] Beatty, G.L. and Russell, C.A. (1940) Tuberculosis of the Flat Bones of the Vault of the Skull. Journal of Bone \& Joint Surgery, 22, 207-210.

[3] Beatty, G.L. and Russell, C.A. (1940) Tuberculosis of the Flat Bones of the Vault of the Skull. The Journal of Bone and Joint Surgery, 22, 207-210.

[4] Shameem, M., Saad, T., Bhargava, R., Ahmad, Z., Fatima, N., Khan, H. and Huda, F. (2009) Frontal Bone Tuberculosis Presenting with Blindness in a 14-Year-Old Girl: A Case Report. Journal of Medical Case Reports, 3, 8220. https://doi.org/10.4076/1752-1947-3-8220

[5] Dharwal, M., Gupta, D.K., Punia, V.P. and Sinha, R.S. (1995) Tuberculous Osteomyelitis of Skull in a Sixty Year Old Female. Journal of the Association of Physicians of India, 43, 575-577.

[6] Sharma, R., Tyagi, I., Kumar, R. and Phadke, R.V. (2000) Tuberculosis of the Skull: A Case Report and Review of the Literature. Neurosurgical Review, 23, 104-106.

[7] Berk, C. (2001) Tuberculosis and the Skull. Journal of Neurosurgery, 94, 146-147. https://doi.org/10.3171/jns.2001.94.1.0146

[8] Inayat, F., Jafar, M.S., Ali, N.S., et al. (2017) Enigma of Extrapulmonary Tuberculosis: Where Do We Stand? Cureus, 9, e1554. https://doi.org/10.7759/cureus.1554

[9] Kaur, A., Kucheria, M., Gupta, R., Thami, G.P. and Kundu, R. (2018) Extensive Multisystemic Disseminated Tuberculosis in an Immunocompetent Patient. Journal of Clinical and Aesthetic Dermatology, 11, 42-46.

[10] Singh, G., Kumar, S., Singh, D.P., Verma, V. and Mohammad, A. (2014) A Rare Case of Primary Tuberculous Osteomyelitis of Skull Vault. Indian Journal of Tuberculosis, 61, 79-83. 